\title{
Mindestsicherung im europäischen Vergleich
}

\section{Thomas Bable}

\section{Einleitung}

Dieser Beitrag analysiert die Funktion der Mindestsicherung für die sozialen Sicherungssysteme in EU- und OECD-Ländern. Dafür wird in Abschnitt 2 Mindestsicherung für den Zweck des internationalen Vergleichs definiert. Anschließend werden die wichtigsten Unterschiede zwischen verschiedenen Ländergruppen bzw. Wohlfahrtsregimen dargestellt (Abschnitt 3). Der darauffolgende Abschnitt 4 behandelt zentrale Entwicklungstendenzen der Mindestsicherung in EU- und OECD-Ländern. Der Fokus ist dabei auf die Entwicklung der Mindestsicherung und ihre Stellung im weiteren sozialen Sicherungssystem gerichtet. Gibt es in dieser Hinsicht ähnliche Entwicklungen in verschiedenen Wohlfahrtsregimen, vielleicht sogar eine Konvergenz?

Der abschließende Abschnitt 5 bündelt die Ergebnisse im Hinblick darauf, welche Rolle die Mindestsicherung in einem effektiven sozialen Sicherungssystem spielen kann und ob sie vielleicht durch ein bedingungsloses Grundeinkommen ersetzbar wäre. Der Beitrag kommt zu dem Schluss, dass Mindestsicherung nur im Rahmen eines insgesamt inklusiven sozialen Sicherungssystems effektiv funktionieren kann. Mindestsicherung kann kein Ersatz für ein ausgebautes Sicherungssystem sein, und auch ein bedingungsloses Grundeinkommen wäre keine gute Alternative. Sozial schwache Bevölkerungsgruppen können auf Dauer nur durch eine bessere allgemeine soziale Sicherung »oberhalb« der Mindestsicherung effektiv geschützt werden.

\section{Definition und Funktion der Mindestsicherung im Wohlfahrtsstaat}

Soziale Mindestsicherung wird in diesem Beitrag als bedürftigkeitsgeprüfte und bedarfsorientierte Leistung an Privatpersonen oder Haushalte definiert, die der 
Sicherung eines sozialen Existenzminimums dient (Bahle/Pfeifer/Wendt 2010; Cantillon/van Mechelen/Schulte 2008; ILO 1942).

Bedürftigkeitsgeprüft bedeutet, dass nur dann Anspruch auf Leistungen besteht, wenn die Eigenmittel der Empfänger:innen (Einkommen oder Vermögen) unterhalb einer definierten Schwelle liegen und es darüber hinaus keine Ansprüche gegenüber Dritten gibt, die den Bedarf decken könnten. Aus der Bedürttigkeitsprüfung folgt, dass die Leistungen der Mindestsicherung residual sind, d.h. nach Berücksichtigung anderer Ansprüche gewährt werden.

Bedarfsorientiert bedeutet, dass die Leistungen die persönlichen Lebensumstände der Empfänger:innen, insbesondere die Haushalts- und Familienkonstellation berücksichtigen. Die Leistungen sind in der Regel gestaffelt nach der Größe und Zusammensetzung der Haushalte und Familien der Empfänger:innen. Sie werden regelmäßig gewährt, d.h. solange der Status der Bedürftigkeit anhält. Punktuelle oder einmalige Leistungen zur Überbrückung bestimmter Notlagen werden hier nicht betrachtet.

Ziel der Leistungen ist es, das soziale Existenzminimum der Empfänger:innen zu sichern. Darunter kann jedoch je nach Kontext und Land Unterschiedliches verstanden werden. Das soziale Existenzminimum umfasst in der Regel die Ausgaben für die notwendigen Dinge des Lebens wie z.B. Nahrung, Kleidung oder Unterkunft. In vielen Ländern werden darüber hinaus Bedürfnisse berücksichtigt, die eine soziale Teilhabe in der jeweiligen Gesellschaft ermöglichen, z.B. Mobilität, Kommunikation oder Teilnahme an kulturellen und Freizeitaktivitäten (vgl. Deeming 2020).

Zur sozialen Mindestsicherung gehört in den meisten Ländern auch der $\mathrm{Zu}-$ gang zu sozialen Diensten wie Gesundheit, Bildung, Kinderbetreuung und Pflege. Diese wichtigen Teilhaberechte variieren zwischen den Ländern und werden in diesem Beitrag nicht betrachtet (vgl. aber die Beiträge von Thomas Gerlinger und Ulf Banscherus in diesem Band).

Im entwickelten Wohlfahrtsstaat hat die soziale Mindestsicherung zwei zentrale Funktionen: Zum einen spannt sie ein letztes Auffangnetz im sozialen Sicherungssystem, zum andern bildet sie das Fundament sozialer Bürgerrechte. Mindestsicherung hat also einen genuin ambivalenten Charakter; sie ist residual und essenziell zugleich (Bahle 2019). Zwischen diesen beiden Polen hat sich die Mindestsicherung historisch entwickelt und bewegt.

$\mathrm{Zu}$ Beginn der staatlichen Sozialpolitik bis zum Ende des 19. Jahrhunderts war die Mindestsicherung in Form der Armenfürsorge von großer Bedeutung. Mit dem Ausbau der sozialen Sicherungssysteme im 20. Jahrhundert gewann der residuale Charakter der Mindestsicherung in Form von Sozialhilfe die Oberhand. Mit der Krise des Wohlfahrtsstaates und dem Anstieg neuer sozialer Risi- 
ken seit den 1980er-Jahren gewann wiederum die essenzielle Funktion der Mindestsicherung als Instrument zur Sicherung fundamentaler sozialer (Bürger-) Rechte an Bedeutung. Allerdings verlief diese Entwicklung in den verschiedenen Wohlfahrtsregimen und Ländern sehr unterschiedlich.

\section{Mindestsicherung in verschiedenen Wohlfahrtsregimen}

Aufgrund der beiden zentralen Merkmale der Mindestsicherung, ihrem residualen und zugleich essenziellen Charakter hinsichtlich sozialer Rechte, ist sie eng mit dem Aufbau der anderen sozialen Sicherungssysteme verknüpft (Bahle/ Hubl/Pfeifer 2011). Zum einen hängt ihre Bedeutung und Funktion davon ab, wie stark soziale Rechte in einer Gesellschaft überhaupt verankert sind. Diese Dimension der sozialen Mindestsicherung betrifft die Schnittstellen zwischen (Sozial-)Staat und Familie sowie zwischen Staat und Markt. Zum andern hängt ihre Funktion davon ab, wie stark die anderen sozialen Sicherungssysteme wie Alterssicherung und Arbeitslosenschutz ausgebaut sind.

Hinsichtlich der zweiten Schnittstelle kann man von einem System kommunizierender Röhren sprechen: Bei gleichbleibenden sozialen Risiken sinkt die Bedeutung der sozialen Mindestsicherung in dem Maße, in dem die Bedeutung der anderen Sicherungssysteme wächst, und umgekehrt. Je mehr soziale Risiken durch die anderen Systeme aufgefangen oder vermindert werden, desto geringer ist die faktische Bedeutung der Mindestsicherung für die Absicherung der Bevölkerung. Daraus sollte jedoch nicht auf eine geringe institutionelle Bedeutung der Mindestsicherung für die Architektur des Wohlfahrtsstaates geschlossen werden, denn ein gutes und festes Fundament ist eine wesentliche Bedingung für die Stabilität des ganzen Hauses.

Auf den ersten Blick gibt es im Ländervergleich eine große und teilweise unübersichtliche Vielfalt an sozialen Mindestsicherungssystemen (vgl. die exzellente Analyse bei Neumann 2016). Tatsächlich existieren in fast allen europäischen Ländern mehrere unterschiedliche Leistungen, die im Sinne der hier verwendeten Definition zur sozialen Mindestsicherung gehören (Bahle/Hubl/ Pfeifer 2011).

In Deutschland z.B. zählen dazu die Grundsicherung für Arbeitsuchende, die Grundsicherung für Ältere und Erwerbsgeminderte, die Sozialhilfe, die Asylbewerberleistungen und die Kriegsopferfürsorge. All diese Leistungen sind bedürttigkeitsgeprüft und bedarfsbezogen und zielen auf die Absicherung eines sozialen Existenzminimums, auch wenn sie sich in der Strenge des Bedürftigkeitstests und beim Inhalt des sozialen Minimums teilweise erheblich voneinander 
unterscheiden. In Deutschland ist die soziale Mindestsicherung also kategorial differenziert, d.h. es gibt unterschiedliche essenzielle soziale Rechte für verschiedene Bevölkerungsgruppen. Dies ist in fast allen europäischen Ländern der Fall.

Trotz dieser Unübersichtlichkeit lassen sich im internationalen Vergleich klare Grundmuster erkennen, die sich aus dem Charakter der jeweiligen Wohlfahrtsregime einerseits und dem Ausbau der sozialen Sicherungssysteme »oberhalb« der Mindestsicherung andererseits ergeben (Bahle 2019; Eardley et al. 1996). Wohlfahrtsregime sind durch einen jeweils typischen Zusammenhang zwischen Staat, Markt und Familie charakterisiert, der für den Ausbau grundlegender sozialer Rechte entscheidend ist. Esping-Andersen (1990) unterscheidet drei klassische Wohlfahrtsregimetypen:

- den sozialdemokratischen Typus mit einer zentralen Rolle des Staates und starken sozialen Rechten (hoher Dekommodifizierung),

- den liberalen Typus mit einer zentralen Rolle des Marktes und schwachen sozialen Rechten (niedriger Dekommodifizierung) und

- den konservativen Typus, der zwischen den beiden erstgenannten liegt.

Leibfried (1992) und Ferrera (2005) identifizieren einen vierten Typus mit schwachen sozialen Rechten und einer großen Bedeutung der (erweiterten) Familie. Für diesen Typus hat sich eine geografische Kennzeichnung eingebürgert: Südeuropa. ${ }^{1}$ Dies gilt auch für einen fünften Typus, der nach dem Zusammenbruch des sozialistischen Systems in Osteuropa entstanden ist und sich von den anderen Typen vor allem durch eine deutlich geringere Entwicklung unterscheidet (Cerami 2006; Nelson 2010).

\section{Südeuropa}

Aus der Perspektive der Mindestsicherung betrachtet, ist zunächst das Verhältnis zwischen Staat und Familie von zentraler Bedeutung. Hierbei sticht vor allem der Unterschied zwischen Südeuropa und den anderen Regimetypen hervor: In den südeuropäischen Ländern übernimmt die Familie nach wie vor eine

1 | In der vergleichenden Forschung werden die Länder üblicherweise wie folgt den verschiedenen Typen zugeordnet:

- liberal: Irland, Kanada, Großbritannien, USA;

- sozialdemokratisch: Dänemark, Finnland, Norwegen, Schweden;

- konservativ: Belgien, Deutschland, Frankreich, Niederlande, Österreich, Schweiz;

- Südeuropa: Griechenland, Italien, Portugal, Spanien.

In einigen Studien werden Finnland dem konservativen, die Schweiz dem liberalen und die Niederlande dem sozialdemokratischen Typus zugeordnet. 
starke Sicherungsfunktion; entsprechend rudimentär sind grundlegende soziale Rechte verankert.

Bis zur Finanz- und Wirtschaftskrise 2008/2009 war Portugal das einzige dieser Länder, das überhaupt ein auf nationaler Ebene institutionalisiertes Mindestsicherungssystem besaß. In Spanien existierten äußerst begrenzte Systeme auf regionaler Ebene, während es in Italien und Griechenland nur sehr wenige lokale oder stark eingeschränkte kategoriale Systeme gab. Nach der Wirtschaftskrise haben diese Länder allerdings Anläufe zu einer auf nationaler Ebene verankerten sozialen Mindestsicherung unternommen. Der Zugang zu diesen Leistungen ist jedoch nach wie vor restriktiv.

\section{Liberale Wohlfahrtsregime}

Im liberalen Wohlfahrtsregime ist die soziale Mindestsicherung hingegen stark verankert, obwohl der Markt hier die Rolle des Staates erheblich einschränkt. Diese starke Verankerung der Mindestsicherung findet sich zumindest in Großbritannien und Irland, den europäischen »Vertretern« dieses Typus. Beide Länder haben soziale Mindestsicherungen, die leicht zugänglich sind und im internationalen Vergleich auch relativ hohe Leistungen gewähren.

Der Grund für diese große Bedeutung der sozialen Mindestsicherung ist, dass die anderen Systeme der sozialen Sicherung hier weniger entwickelt sind. Die Mindestsicherung übernimmt also wesentliche Teile der wohlfahrtsstaatlichen Funktion. Da es sich um zentrale Elemente der sozialen Sicherung handelt, sind die Mindestleistungen auch entsprechend institutionalisiert und bilden ein festes Fundament sozialer Rechte.

Die beherrschende Rolle des Marktes wirkt sich bei diesem Typus also erst auf der zweiten Ebene aus. Diese betrifft nicht die grundsätzliche Frage wohlfahrtsstaatlicher Verantwortung, sondern die Frage der Aufteilung sozialer Risiken im sozialen Sicherungssystem zwischen Sicherung des Lebensstandards einerseits und Grund- und Mindestsicherung andererseits. Der Schwerpunkt im liberalen Typus liegt klar auf den letztgenannten Elementen. Dies gilt jedoch nicht für die USA, wo es bis heute kein starkes Fundament für soziale Bürgerrechte gibt.

\section{Sozialdemokratische Wohlfahrtsregime}

Im sozialdemokratischen Typus hat der Staat die beherrschende Rolle. Dies führt zu einer faktisch rudimentären Funktion der Mindestsicherung im Wohlfahrtsstaat, denn die anderen Systeme der sozialen Sicherung sind hoch entwickelt. Der Zugang zu diesen allgemeinen Systemen ist inklusiv, viele davon sind universal auf die ganze Bevölkerung gerichtet, und die Leistungen liegen deutlich 
über dem Existenzminimum. Aus diesem Grund fallen nur wenige Menschen, hauptsächlich solche mit persönlicher Migrationserfahrung, ins letzte Auffangnetz der sozialen Mindestsicherung.

Deshalb ist der Bezug von Mindestsicherung in diesen Ländern tatsächlich häufig ein klares Indiz für soziale Exklusion. Ein weiterer Indikator dafür sind auch die im internationalen Vergleich niedrigen Leistungen der Mindestsicherung. Nur die dänischen und finnischen Leistungen liegen im oberen Drittel der europäischen Länder, allerdings nicht in den Systemen für zugewanderte Menschen mit kürzerem Aufenthalt. In Schweden und Norwegen sind die Leistungen eher unterdurchschnittlich (Frazer/Marlier 2016; OECD 2021).

\section{Konservative Wohlfahrtsregime}

Im konservativen Regimetyp ist die Situation in den verschiedenen Ländern deutlich heterogener. Grundsätzlich liegt dieser Typus zwischen den drei anderen. Im Vergleich zu den südeuropäischen Ländern sind soziale Risiken weit stärker durch den Wohlfahrtsstaat als durch die Familie abgesichert, d.h. soziale Rechte sind stärker verankert. In allen Ländern des konservativen Wohlfahrtsregimetypus existieren insofern Mindestsicherungssysteme, die aber wie im deutschen Fall zumeist kategorial differenziert sind (Hubl/Pfeifer 2013). Es gibt also keine einheitliche Mindestsicherung für alle Bürger:innen oder Bewohner:innen eines Landes.

Nicht nur werden verschiedene Gruppen unterschiedlich behandelt, einige Bevölkerungsgruppen sind de facto von der Mindestsicherung ausgeschlossen, in Deutschland z. B. Studierende. Für diese Gruppe ist nach wie vor primär die Herkunftsfamilie zuständig, erst in zweiter Linie greift das BAföG, das man jedoch aufgrund seiner spezifischen Merkmale nicht zu den Mindestsicherungsleistungen zählen kann.

Hinsichtlich des Ausbaus anderer Systeme der sozialen Sicherung liegen die Länder des konservativen Typus zwischen dem liberalen und dem sozialdemokratischen Typus. Die Systeme der Alters- und Arbeitslosenversicherung sind z. B. stärker ausgebaut als in den liberalen Ländern, aber weniger als in den sozialdemokratischen. Auch die Familienpolitik bewegt sich etwa im Mittelfeld zwischen diesen Typen. Ältere Menschen, Arbeitslose und Alleinerziehende sind in fast allen Ländern die Hauptgruppen, die auf Mindestsicherung angewiesen sind. Da sich die konservativen Länder teilweise stark in den sozialen Sicherungssystemen für diese drei Gruppen unterscheiden, weisen auch ihre Mindestsicherungssysteme unterschiedliche Profile auf (vgl. für ältere Menschen Goedemé und Marchal 2016). 
In den Niederlanden z. B. gibt es aufgrund der hohen staatlichen Grundrenten für alle Wohnbürger:innen kaum ältere Menschen unter den Bezieher:innen von Mindestsicherung. In Deutschland wiederum sind aufgrund der Hartz-Reformen überdurchschnittlich viele Arbeitslose auf Mindestsicherung angewiesen. Hierzulande ist auch der Anteil der Alleinerziehenden, die von Mindestsicherung leben, sehr hoch, unter anderem weil die Familienpolitik das Problem der Vereinbarkeit von Familie und Beruf nicht effektiv gelöst hat.

Trotz dieser Variationen innerhalb der Gruppe der konservativen Länder spielt die Mindestsicherung in allen eine deutlich geringere Rolle als in den liberalen Ländern (weil die sozialen Sicherungssysteme stärker entwickelt sind), aber eine größere als in den sozialdemokratischen Ländern (weil die Systeme weniger inklusiv sind). In allen Ländern des konservativen Typus zeigen sich Lücken im sozialen Sicherungssystem, wenn auch in unterschiedlichen Bereichen, die vom letzten Netz der Mindestsicherung aufgefangen werden müssen.

\section{Osteuropa}

Die osteuropäischen Länder zeigen wiederum ein eigenes Profil. Dort sind die Mindestsicherungen in der Regel auf einem sehr niedrigen Niveau verankert, weshalb nur die extrem Bedürftigen überhaupt Leistungen bekommen (Bahle/Hubl/ Pfeifer 2011; Frazer/Marlier 2016). Auf der anderen Seite haben viele dieser Länder relativ umfassende, wenngleich ebenfalls auf niedrigem Niveau angesiedelte soziale Sicherungssysteme. Strukturell ähneln die meisten osteuropäischen Länder den konservativen Wohlfahrtsstaaten, aber auf deutlich niedrigerem Niveau. In den südosteuropäischen Ländern spielt außerdem ähnlich wie in Südeuropa die Familie noch eine große Rolle. Insgesamt haben diese Länder also eher rudimentäre Mindestsicherungssysteme, die nur wenige Bedürttige tatsächlich erreichen.

Die bisherige Analyse konzentrierte sich auf die Rolle der Mindestsicherung im Gesamtgefüge der sozialen Sicherung in verschiedenen Wohlfahrtsregimen. Dabei stand der Aspekt der Inklusion und des Ausbaus sozialer Rechte im Mittelpunkt. Eine weitere zentrale Frage ist, wie hoch die Leistungen der Mindestsicherung sind und ob sie Armut effektiv bekämpfen können. Die Antwort darauf fällt in fast allen Ländern eindeutig negativ aus: Die Höhe der Leistungen reicht in der Regel nicht aus, um die betroffenen Menschen aus der relativen Einkommensarmut zu befreien, zumindest dann nicht, wenn man die Armutsschwelle bei 60 Prozent des verfügbaren äquivalenzgewichteten Medianeinkommens im jeweiligen Land festlegt (siehe Abbildung 1; vgl. auch Bahle 2019; Frazer/Marlier 2016). 
Abb. 1: Garantiertes Mindesteinkommen* in Relation zum nationalen äquivalenzgewichtetem Medianeinkommen (alleinstehende Person ohne Beschäftigung 2020; in Prozent)

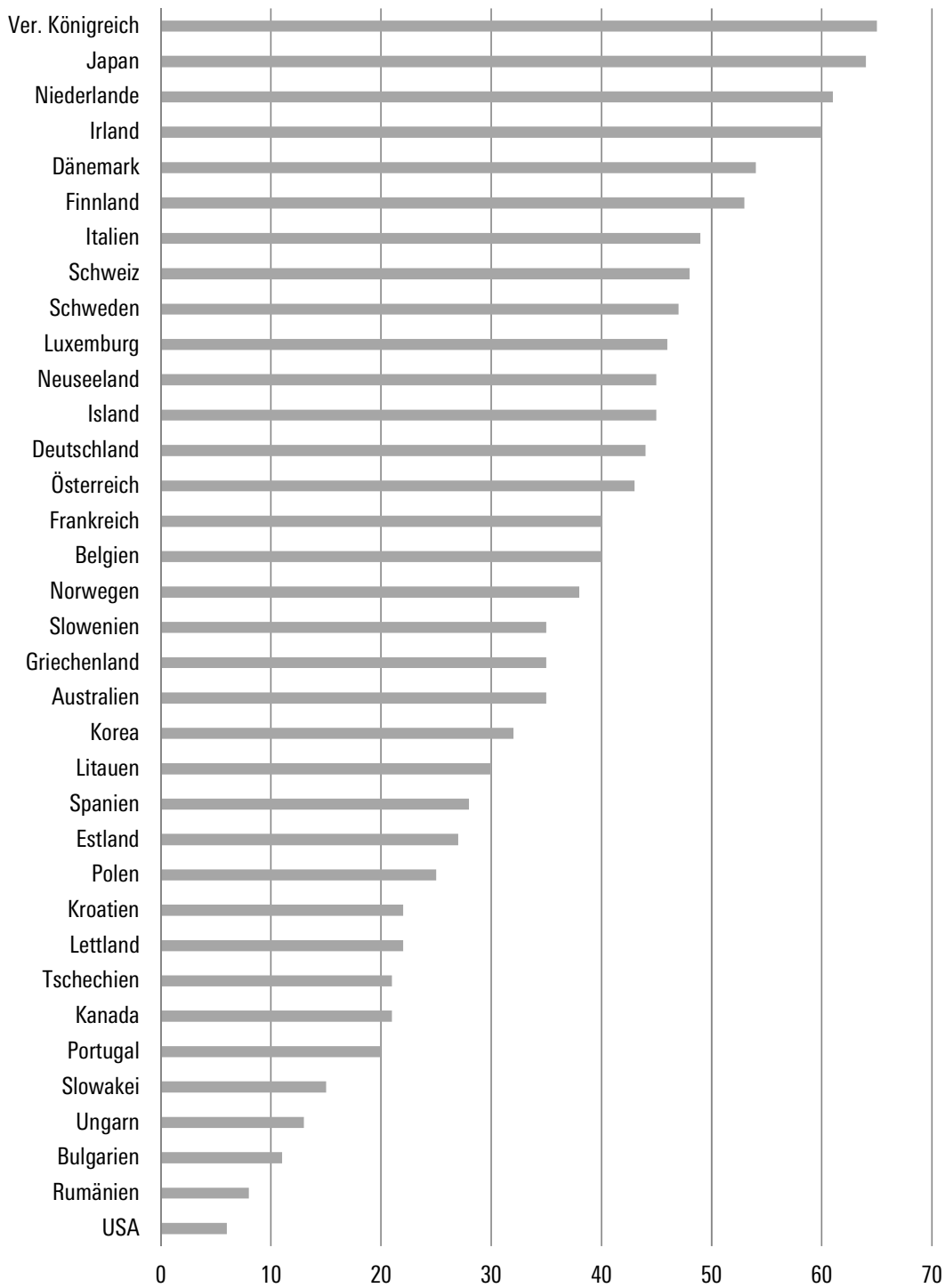

*Anmerkung: garantiertes Mindesteinkommen einschließlich Wohnleistungen Quelle: OECD 2021 (eigene Darstellung). 
Selbst bei einer strengeren Schwelle von 50 Prozent des Medianeinkommens liegen die Leistungen der Mindestsicherung in den meisten Ländern darunter. Nur in Finnland, Dänemark, den Niederlanden und Japan sowie - vielleicht überraschenderweise - in Irland und Großbritannien liegen die Leistungen darüber.

Die Werte schwanken je nach Familienkonstellation; in Deutschland und Polen z. B. erhalten Paare mit Kindern relativ zum jeweiligen äquivalenzgewichteten Medianeinkommen deutlich höhere Leistungen. Die Zahlen für Großbritannien schwanken außerdem stark, je nachdem welche Wohnkosten in den Modellanalysen veranschlagt werden (vgl. die Analysen von Bradshaw und Marchal in Frazer/Marlier 2016).

Die fünf europäischen Länder, in denen die Werte über 50 Prozent liegen, sind auch diejenigen, in denen die Idee der Grundsicherung generell eine wichtige Rolle spielt. Mindestsicherung ist also offensichtlich vor allem dann mit höheren Leistungen verbunden, wenn soziale Grundrechte insgesamt stark in der Gesellschaft verankert sind, sei es auf eher »liberalem« Weg (wie z. B. in Großbritannien oder Irland) oder sozialdemokratisch gefärbt (wie in Dänemark).

Dennoch ist der klare Befund aus dem internationalen Vergleich, dass Mindestsicherung in kaum einem Land relative Armut tatsächlich effektiv vermindert (van Mechelen/Marchal 2013). Unbestritten ist hingegen, dass sie Armutslagen lindert und Deprivation relativ erfolgreich verhindern kann (Nelson 2010).

Ob die Mindestsicherung den betroffenen Menschen auch Chancen vermitteln kann, um sich aus eigenen Kräften aus dieser prekären Lage zu befreien, ist eine weitere zentrale Frage, die hier nicht systematisch analysiert werden kann. Es kommt dabei entscheidend auf die Verknüpfung der Mindestsicherung mit anderen gesellschaftlichen Bereichen an, vor allem mit dem Arbeitsmarkt, der Familienpolitik, dem Gesundheits- und dem Bildungssystem.

In den liberalen Ländern, vor allem auch in den USA, sind die Mindestsicherungssysteme sehr stark auf die Aufnahme einer Erwerbstätigkeit gerichtet. In den USA z. B. ist der Earned Income Tax Credit (EITC), eine Steuergutschrift für Niedrigverdiener:innen, seit vielen Jahren das größte Wohlfahrtsprogramm überhaupt. In Großbritannien ist die Jobseeker Allowance ebenfalls mit starken Anreizen zur Aufnahme einer Erwerbsarbeit verbunden, auch im Niedriglohnbereich.

In Deutschland zielt die Grundsicherung für Erwerbsfähige in die gleiche Richtung. Ob damit jedoch nur eine Beschäftigung im Niedriglohnbereich erzielt wird oder es darüber hinaus einen »Fahrstuhleffekt « nach oben gibt, ist umstritten und hängt sicher auch von der konjunkturellen Lage auf dem Arbeitsmarkt ab. 
Der Zusammenhang mit dem Gesundheitssystem ist ebenfalls wesentlich. In Deutschland beispielsweise sind viele Menschen, die Arbeitslosengeld II beziehen, de facto aus gesundheitlichen Gründen nicht voll erwerbsfähig; umso wichtiger ist der Zugang zu medizinischen und sozialen Diensten für die Betroffenen. In den USA sind jedoch viele ärmere Menschen trotz der Reform von 2010 immer noch ohne Krankenversicherungsschutz, was ihre ohnehin prekäre Lage, die ja häufig mit starken gesundheitlichen Einschränkungen einhergeht, zusätzlich verschärft (vgl. Case/Deaton 2020). Bildung schließlich ist vor allem in der Generationenfolge ein Schlüssel zum Ausstieg aus Armutslagen.

Der internationale Vergleich zeigt somit erhebliche Unterschiede in Ausgestaltung und Bedeutung der Mindestsicherung zwischen den Ländern. Es lassen sich einerseits Zusammenhänge mit den verschiedenen Wohlfahrtsregimen erkennen, andererseits spielt auch die Entwicklung der sozialen Sicherungssysteme in den verschiedenen Bereichen eine Rolle, die zum Teil auch innerhalb der Typen variiert. Mindestsicherung korrespondiert eng mit den Systemen der Alterssicherung, des Arbeitslosenschutzes und der Familienpolitik, um nur die wichtigsten Bereiche und Risikogruppen zu nennen.

Auch die Frage, wie Migrant:innen und Flüchtlinge in den jeweiligen Systemen der verschiedenen Länder behandelt werden, wird zunehmend wichtiger, weil der Anteil dieser Bevölkerungsgruppe in allen Ländern zunimmt. Hierbei wird deutlich, dass Flüchtlinge und Asylbewerber:innen in fast allen Ländern deutlich weniger soziale Rechte haben als andere Gruppen (Hubl/Pfeifer 2013). Dies gilt gerade auch in den ansonsten generösen nordischen Wohlfahrtsstaaten.

Die Mindestsicherung allein kann also kein effektives Mittel zur Bekämpfung von Armut sein. Dafür sind die Systeme zu heterogen und lückenhaft, in manchen Ländern generell zu wenig ausgebaut. Darüber hinaus sind die Leistungen deutlich zu niedrig, um die Menschen aus Armut zu befreien. Damit Betroffene die Chance erhalten, sich selbst aus einer solchen Lage befreien zu können, ist vor allem der Zugang zu anderen Systemen wie Arbeit, Gesundheit und Bildung zentral. Das letzte Auffangnetz allein kann somit kein wirkliches Fundament für den Wohlfahrtsstaat sein; es ist aber Teil einer Gesamtarchitektur, die für ein stabiles Haus nötig ist.

\section{Wandel der Mindestsicherung}

Im vorangehenden Abschnitt 3 standen die Unterschiede zwischen den Mindestsicherungssystemen der verschiedenen Wohlfahrtsregimetypen und Länder im Mittelpunkt. In diesem Abschnitt geht es um den Wandel der Mindestsiche- 
rung über die Zeit: Welche Trends lassen sich hierbei beobachten? Gibt es große internationale Unterschiede oder überwiegen gemeinsame Entwicklungen?

An dieser Stelle ist eine systematische Analyse vieler Länder über die Zeit hinweg nicht möglich; auch gibt es dazu kaum umfassende Langzeitstudien. Die meisten vergleichenden Analysen betrachten einen bestimmten Zeitpunkt (Querschnitt), einen bestimmten Aspekt über die Zeit hinweg (z. B. die Höhe der Leistungen; vgl. Marchal/Marx/van Mechelen 2014; Nelson 2010) oder nur wenige oder einzelne Länder im Zeitverlauf. Dennoch lassen sich hier einige wichtige Befunde festhalten.

Insgesamt haben sich die Mindestsicherungssysteme in den verschiedenen Ländern und Wohlfahrtsregimen einander etwas angenähert, aber die grundlegenden Unterschiede blieben erhalten. Es fand also keine Konvergenz statt. In den südeuropäischen Ländern erfuhr die Mindestsicherung seit der Finanz- und Wirtschaftskrise 2008/2009 einen Ausbauschub (vgl. Matsaganis 2017); diese Länder holten ihren Rückstand in dieser Hinsicht also auf. In vielen - vor allem konservativen - Ländern fand ein Umbau der sozialen Sicherungssysteme mit teilweise massiven Folgen für die Mindestsicherung statt.

Dies war vor allem in Deutschland der Fall. Hierdurch gewann die Mindestsicherung für die soziale Sicherung insgesamt an Bedeutung. In fast allen Ländern konnten die Leistungen der Mindestsicherung jedoch nicht mit dem allgemeinen Einkommenszuwachs Schritt halten (Marchal/Marx/van Mechelen 2014). Dieser Befund ist von zentraler Bedeutung für die Frage, welche Rolle die Mindestsicherung in Zukunft innerhalb der sozialen Sicherung spielen sollte (vgl. Marx/Nelson 2013).

Die Folgen der Finanz- und Wirtschaftskrise waren vor allem in den südeuropäischen Ländern gravierend. Der Anstieg der Arbeitslosigkeit bei gleichzeitig wachsendem Druck auf die Staatsfinanzen stürzte viele Menschen in eine äußerst prekäre Lage. Davon betroffen waren vor allem junge Menschen und Erwerbstätige, während die Älteren zunächst relativ gut abgesichert waren. In vielen Ländern fehlte ein adäquates soziales Sicherungssystem für diese Gruppen, und die Familien waren mit dieser Aufgabe hoffnungslos überfordert.

Die Einführung einer effektiven sozialen Mindestsicherung erschien somit als Gebot der Stunde, darüber hinaus als eine kostengünstige Alternative zu anderen Instrumenten. Dennoch dauerte es einige Jahre, bis Griechenland und Italien zum ersten Mal tatsächlich Mindestsicherungssysteme auf nationaler Ebene einführten. Dies geschah erst, als die schlimmsten Folgen der Krise schon abgeebbt waren.

Dafür gab es zum einen politische Gründe - im Falle Griechenlands auch im Zusammenhang mit dem nationalen Widerstand gegen die Politik der Troika 
aus EU, EZB und IWF -, zum anderen aber auch gewichtige systemische Ursachen. Mindestsicherungssysteme sind mit erheblichem Informationsbedarf und Verwaltungsaufwand verbunden. Nicht zuletzt hängt ihre Wirksamkeit vom Vertrauen der Menschen in den Staat und vom Vertrauen des Staates in die Bürger:innen ab. Insbesondere die Bedürftigkeitsprüfung und die Bedarfsfeststellung erfordern effektive administrative Strukturen und Handlungskapazitäten des Staates sowohl auf nationaler als auch auf lokaler Ebene.

Diese Voraussetzungen waren und sind in einigen südeuropäischen Ländern nicht gegeben. Weder funktioniert die Kooperation zwischen Zentralstaat, Regionen und Kommunen noch sind Finanz-, Arbeits- und Sozialverwaltung miteinander koordiniert. Selbst in Deutschland mit seiner grundsätzlich gut funktionierenden öffentlichen Verwaltung hat die Zusammenlegung von Arbeits- und Sozialbehörden in den Jobcentern größere Verwerfungen hervorgerufen, die bis heute nachwirken. Gerade eine effektive und einheitliche Mindestsicherung ist organisatorisch und kulturell höchst voraussetzungsvoll. Erst langsam wurden in den betroffenen südeuropäischen Ländern die dafür notwendigen administrativen Bedingungen geschaffen.

Die kulturellen Grundlagen für Vertrauen und für das Sozialkapital einer Gesellschaft lassen sich ohnehin nur schwer beeinflussen. Es ist deshalb kein $\mathrm{Zu}-$ fall, dass die Bewegung Cinque Stelle in Italien massiv für ein staatlich garantiertes Grundeinkommen anstelle einer Mindestsicherung in klassischer Form eintrat, denn ein solches Grundeinkommen wäre weit weniger anspruchsvoll. Dies zeigen auch Erfahrungen aus ärmeren Schwellen- und Entwicklungsländern, wo statt einer allgemeinen Mindestsicherung meistens kategoriale Programme für bestimmte Bevölkerungsgruppen wie Ältere oder Kinder (sogenannte »Demogranten«) eingeführt wurden, weil diese Programme leichter zu administrieren und mit weniger Missbrauchsmöglichkeiten verbunden sind (Leisering 2019).

Italien und Griechenland haben mit der Einführung ihrer Programme in den 2010er-Jahren teilweise zum europäischen Standard aufgeschlossen. In anderen europäischen Ländern wurden die sozialen Sicherungssystem und mit ihnen die Mindestsicherung zum Teil gründlich umgebaut. Dieser Prozess setzte in den westeuropäischen Ländern in den 1990er-Jahren ein, in den osteuropäischen Ländern mussten die Systeme nach dem Fall des Kommunismus überhaupt erst neu aufgebaut werden, denn in der sozialistischen Gesellschaft gab es (angeblich) weder Armut noch Arbeitslosigkeit.

In den osteuropäischen Ländern wurden im Zuge der Transformation tatsächlich in der Regel umfassende (universelle) Mindestsicherungen eingeführt, wenngleich auf sehr niedrigem Sicherungsniveau. In den westeuropäischen Län- 
dern hingegen überwog ein Trend zur Differenzierung, also die kategoriale Aufsplitterung ehemals einheitlicher Mindestsicherungen.

Paradigmatisch dafür ist der deutsche Fall. Aus der 1962 eingeführten umfassenden Sozialhilfe wurden im Laufe der Zeit vier verschiedene Systeme mit unterschiedlichen Regeln und Zielen: Leistungen für Asylbewerber:innen und Flüchtlinge (1993), Grundsicherung für Ältere und Erwerbsgeminderte (2003) und Grundsicherung für Erwerbsfähige (2005); daneben fungiert die »alte« Sozialhilfe weiter als »Residuum der Residuen «. In der Diskussion ist derzeit eine Grundsicherung für Kinder (allerdings meistens nicht bedürftigkeitsgeprüft!). Diese würde dem Tableau ein weiteres Feld hinzufügen, hätte darüber hinaus aber auch Rückwirkungen auf die bereits existierenden Systeme, insbesondere auf dasjenige für Erwerbsfähige.

Wie auch immer, diese Entwicklung folgt einer klaren Logik der zunehmenden Bearbeitung sozialer Risiken durch Elemente der Mindestsicherung. Mindestsicherung wird auf diese Weise zu einem Massenprogramm, zu einem zentralen Element der sozialen Sicherung in allen Bereichen (siehe hierzu auch den Beitrag von Gerhard Bäcker in diesem Band). Etwas pointiert könnte man sagen, dass wir damit in Deutschland auf dem Weg in eine durchgehend zweistufige Landschaft der sozialen Sicherung in allen Feldern sind: Alterssicherung (plus Grundsicherung für Ältere), Arbeitslosensicherung (plus Grundsicherung für Erwerbsgeminderte) und Familienpolitik (plus Grundsicherung für Kinder?).

Die zentrale Frage ist, ob sich dadurch tatsächlich der Schutz benachteiligter Gruppen in den einzelnen Bereichen verbessern lässt oder ob diese Entwicklung nicht letztlich in einen Sozialstaat für zwei Klassen mündet, wobei die benachteiligten Gruppen weiter zurückfallen.

Zeigt sich in verschiedenen Ländern zum Teil ein Ausbau, zum Teil ein Umbau der Mindestsicherungssysteme, so lässt sich generell für fast alle Länder sagen, dass die Leistungen der Mindestsicherung über die Zeit hinweg bestenfalls stagnieren, in weiten Teilen sogar im Vergleich zu anderen Maßzahlen wie Durchschnittseinkommen oder Preisniveau sinken. Überhaupt bringen sie die Empfänger:innen nur in wenigen Ländern knapp über die Armutsschwelle (siehe Abschnitt 3).

Diese Entwicklung offenbart, dass Mindestsicherung kein sonderlich leistungsfähiges oder inklusives Instrument der sozialen Sicherung ist. Sie mildert Armutslagen, schafft aber kein gutes Fundament für den Wohlfahrtsstaat. Alarmieren müssen in diesem Zusammenhang Überlegungen und Ideen, die Grundsicherung quasi zum herrschenden Prinzip für den Sozialstaat machen zu wollen. 
Im Ländervergleich lässt sich zur Entwicklung der Mindestsicherung also folgendes Fazit festhalten: Beginnend mit der britischen Social Assistance von 1945 haben mit Griechenland (2017) und Italien (2019) nach über siebzig Jahren nunmehr alle europäischen Länder eine Form der Mindestsicherung auf nationaler Ebene eingeführt (vgl. Neumann 2016). Der größte Schub in diese Richtung ergab sich durch die um 1990 geschaffenen Systeme im Zuge der Transformation in Osteuropa.

Trotz dieser allgemeinen Entwicklung bleiben große Unterschiede in der Ausgestaltung der Mindestsicherung zwischen den Ländern erhalten, die in groben Zügen den verschiedenen Wohlfahrtsregimen entsprechen. Mindestsicherung ist Teil der Gesamtarchitektur des Wohlfahrtsstaates und nur im Zusammenhang mit den anderen Systemen der sozialen Sicherung zu verstehen. Wollte man zentrale gemeinsame Entwicklungstrends ausmachen, gäbe es dafür zwei Punkte:

- In fast allen Ländern wurden die Mindestsicherungen stärker kategorial differenziert.

- In fast allen Ländern hinken die Leistungen weiter hinter der allgemeinen Einkommensentwicklung her.

Mindestsicherung wird also weniger universal und weniger effektiv.

\section{Von der Mindestsicherung zum bedingungslosen Grundeinkommen?}

Die Analyse ergab große Unterschiede, aber auch einige Gemeinsamkeiten in den Mindestsicherungssystemen europäischer Länder. In dieser abschließenden Diskussion und Bewertung der zentralen Ergebnisse möchte ich auf zwei Fragen eingehen:

- Ist die Einführung von Mindestsicherungen in verschiedenen Bereichen ein adäquates Instrument sozialer Sicherung?

- Wäre ein bedingungsloses Grundeinkommen eine gute Alternative zur Mindestsicherung?

An dieser Stelle kann und soll keine ausführliche Diskussion über das Für und Wider eines bedingungslosen Grundeinkommens erfolgen, vielmehr geht es um die Frage, ob ein solches Instrument dazu geeignet wäre, die dargelegten Defizite der Mindestsicherung zu beheben. 


\section{Ist die Einführung von Mindestsicherungen in verschiedenen Bereichen ein adäquates Instrument sozialer Sicherung?}

Auf den ersten Blick erscheinen Mindestsicherungen in den verschiedenen Bereichen des Wohlfahrtsstaates als ein geeignetes Instrument, um die Position sozial schwacher Gruppen im Gefüge der sozialen Sicherung zu verbessern. Die Grundsicherung für Ältere ist ein Schutz für diejenigen, deren Einkommen im Alter nicht zum Leben ausreicht.

Doch wir sprechen hier von einer Mindestsicherung, d.h. die Leistungen sind bedürftigkeitsgeprüft und bedarfsorientiert. Das Problem ist hierbei vor allem das erste Merkmal. De facto wurde durch die Einführung einer solchen kategorialen Mindestsicherung für Ältere ein zweistufiges System der öffentlichen Alterssicherung geschaffen - ohne hier auf die privaten oder betrieblichen Formen »oberhalb« der öffentlichen Säulen eingehen zu wollen. Diese Dualität führt zu zwei großen Gefahren:

- Erstens entsteht eine dauerhafte Spaltung zwischen den Sicherungssystemen und Interessen der Durchschnittsverdiener:innen einerseits und der sozial Benachteiligten andererseits. Dadurch verlieren die Leistungen im bedürftigkeitsgeprüften System über die Zeit an Akzeptanz, Legitimität und Wert. Dieses »Paradoxon der Umverteilung« (Korpi/Palme 1998) ist ein zentraler Befund der vergleichenden Wohlfahrtsstaatsforschung: Je stärker sich soziale Programme auf ärmere Schichten konzentrieren, desto schwächer wird deren politische und soziale Unterstützungsbasis.

- Die zweite Gefahr schließt unmittelbar daran an: Zwar wird die Mittelschicht »ihr« einkommensbezogenes und nicht bedürftigkeitsgeprüftes Alterssicherungssystem verteidigen, aber die Existenz eines darunterliegenden letzten Auffangnetzes der Mindestsicherung erleichtert ein Absenken des Sicherungsniveaus im allgemeinen Rentensystem - denn sollte das Niveau im Einzelfall unter eine existenzbedrohende Schwelle absinken, gibt es ja für die Betroffenen einen Schutz. Potenziell ist also in einem zweistufigen System die »untere« Schicht im »oberen« System immer der Gefahr des Abgleitens ins »untere« System ausgesetzt.

Das System kann dadurch leicht ins Rutschen kommen. Da im unteren System eine Bedürftigkeitsprüfung greift, müssen die Betroffenen aber vor Leistungsbeginn aus diesem System noch ihre eigenen Reserven liquidieren. Tatsächlich schreckt diese Aussicht - und andere Gründe wie Scham oder Informationsmangel - schon heute viele Leistungsberechtigte davon ab, Mindestsicherung zu beantragen. Aus der Perspektive des Risikoschutzes für sozial schwächere Gruppen wäre deshalb ein einstufiges System der Ab- 
sicherung mit dortiger Einführung eines Mindestniveaus die weit effektivere Lösung, also beispielsweise eine Mindestrente innerhalb der allgemeinen Alterssicherung.

Dieselben Argumente lassen sich ebenso für alle anderen Bereiche des Wohlfahrtsstaates anführen. Inklusion ist für sozial Schwächere immer besser als Exklusion und "Sonderbehandlung« in spezifischen Programmen und Einrichtungen. Alleinerziehende profitieren z.B. mehr von einer hoch entwickelten Familienpolitik als von speziell für sie entworfenen Programmen. Dies belegt der Vergleich zwischen den skandinavischen und den angelsächsischen Ländern eindrücklich. Ärmere Menschen profitieren mehr von einer guten inklusiven Gesundheitsversorgung als von speziellen Programmen, wie das Beispiel der USA zeigt. Kinder aus sozial benachteiligten Schichten haben größere Chancen in Kindergärten und Schulen, die Kindern aller Gesellschaftsschichten offenstehen, als in speziellen Einrichtungen.

Jede Form der Mindestsicherung birgt die Gefahr einer wachsenden sozialen Spaltung. Lücken in der sozialen Absicherung sollten deshalb bevorzugt innerhalb und nicht unterhalb bestehender allgemeiner Systeme geschlossen werden.

\section{Wäre ein bedingungsloses Grundeinkommen eine gute Alternative zur Mindestsicherung?}

Daher stellt sich die Frage, ob die Defizite der Mindestsicherung nicht auf einen Schlag durch ein allgemeines bedingungsloses Grundeinkommen beseitigt werden könnten. Tatsächlich hat diese Idee auf den ersten Blick Charme, würde ein solches Instrument doch vieles vereinfachen und dazu noch den ungeliebten Bedürftigkeitstest überflüssig machen. Blickt man nur auf die Seite der Mindestsicherung, wird man dem »alten« System wahrscheinlich tatsächlich keine Träne nachweinen.

Doch beim bedingungslosen Grundeinkommen besteht die Gefahr auf der anderen Seite der Ungleichung, denn durch dieses könnten die etablierten sozialen Sicherungssysteme insgesamt unter Druck geraten bzw. sogar scheinbar überflüssig werden. In einigen radikalen Vorschlägen zum bedingungslosen Grundeinkommen geht es tatsächlich um nichts anderes als um die Abschaffung des Sozialstaats. Hiermit sind aber zwei große Gefahren verbunden:

- Erstens gilt zumindest mittel- bis langfristig auch für das bedingungslose Grundeinkommen das Paradoxon der Umverteilung. Die Mehrheit der Bevölkerung, deren Einkommen über dem Grundeinkommen liegt, hat keinerlei Interesse daran, dieses Grundeinkommen langfristig abzusichern oder gar 
weiterzuentwickeln. Es würde also auch hier de facto eine klare und noch dazu tiefe Spaltung in der Gesellschaft entstehen, wobei die tatsächlich Bedürftigen die Verlierer:innen sein dürften.

- Zweitens würde der bisher stärkste Pfeiler der sozialen Sicherung zerstört, der für die Stabilität und Legitimität des Wohlfahrtsstaates bisher von entscheidender Bedeutung war: die Bindung und Begründung sozialer Leistungen durch klar identifizierte und anerkannte soziale Risiken. Dieser Zusammenhang ist geradezu der Beginn der modernen staatlichen Sozialpolitik, als bestimmte Lebensrisiken, die mit dem Aufkommen der Industrialisierung einhergingen, als soziale Risiken anerkannt wurden, für die spezifische Sicherungsinstitutionen wie Alters- und Krankenversicherung geschaffen wurden - gerade die Abkehr vom Fürsorgeprinzip hat den Aufstieg des Wohlfahrtsstaates ermöglicht.

Ein bedingungsloses Grundeinkommen wäre ein radikaler Schritt zurück und ein Bruch mit dem Grundprinzip der kollektiven Absicherung sozialer Risiken zugunsten einer radikal individualistischen Sichtweise von Selbstverantwortung des Individuums. Dies erscheint aufgrund der offensichtlichen Zunahme sozialer Risiken, auch bedingt durch Migration und die Digitalisierung der Arbeitswelt, als gefährlicher Weg, bei dem die sozial Schwachen nur verlieren können.

Der große Vorteil, den ein bedingungsloses Grundeinkommen aus Sicht einer effektiven sozialen Sicherung tatsächlich haben könnte, wäre, dass sich die Lücken im System sozusagen auflösen würden. Dass diese Lücken bestehen und zum Teil wachsen, ist offensichtlich. Doch ein besserer, wenngleich mühsamerer Weg zu dem Ziel, diese Lücken zu schließen, liegt innerhalb der bestehenden Systeme der sozialen Sicherung.

In Deutschland würde dies z. B. bedeuten, dass Selbstständige und Niedrigverdiener:innen in die allgemeinen Systeme integriert werden. Alleinerziehende sollten durch besser ausgebaute Maßnahmen zur Vereinbarung von Familie und Beruf unterstützt werden. Der Weg zu einer effektiveren sozialen Sicherung führt nicht über Exklusion, sondern über Inklusion. Die individualistisch inspirierte Idee eines bedingungslosen Grundeinkommens ist ein Irrweg - soziale Mindestsicherung kann ihre essenzielle Funktion als Fundament sozialer Bürgerrechte nur dann effektiv erfüllen, wenn sie zugleich residual innerhalb eines entwickelten sozialen Sicherungssystems ist.

Insofern löst sich die zu Beginn dieses Beitrags festgestellte konzeptionelle Ambivalenz der Mindestsicherung als Fundament des Wohlfahrtsstaates und letztem Auffangnetz der sozialen Sicherung in der Praxis auf: In einer differen- 
zierten und sich ständig wandelnden Gesellschaft können grundlegende soziale Rechte nur im Rahmen differenzierter Sicherungssysteme für alle Gruppen der Bevölkerung nachhaltig gesichert werden.

\section{Literatur}

Bahle, T. (2019): Soziale Mindestsicherung, in: Obinger, H./Schmidt, M.G. (Hrsg.): Handbuch Sozialpolitik, Heidelberg, S. 761-782.

Bahle, T./Hubl, V./Pfeifer, M. (2011): The Last Safety Net. A Handbook of Minimum Income Protection in Europe, Bristol.

Bahle, T./Pfeifer, M./Wendt, C. (2010): Social assistance, in: Castles, F./Leibfried, S./Lewis, J./Obinger, H./Pierson, C. (Hrsg.): The Oxford Handbook of the Welfare State, Oxford, S. 448-461 (eine aktualisierte Ausgabe erscheint 2021).

Cantillon, B./van Mechelen, N./Schulte, B. (2008): Minimum income policies in old and new member states, in: Alber, J./Fahey, T./Saraceno, C. (Hrsg.): Handbook of Quality of Life in the Enlarged European Union, London, S. 218-234.

Case, A./Deaton, A. (2020): Deaths of Despair and the Future of Capitalism, Princeton.

Cerami, A. (2006): Social Policy in Central and Eastern Europe. The Emergence of a New European Welfare Regime, Berlin.

Deeming, C. (Hrsg.) (2020): Minimum Income Standards and Reference Budgets. International and Comparative Policy Perspectives, Bristol.

Eardley, T./Bradshaw, T./Ditch, J./Gough, I./Whiteford, P. (1996): Social Assistance in OECD Countries. Synthesis Report, Department of Social Security, Research Report No. 46, London.

Esping-Andersen, G. (1990): The Three Worlds of Welfare Capitalism, Cambridge.

Ferrera, M. (2005): Welfare states and social safety nets in Southern Europe: an introduction, in: Ferrera, M. (Hrsg.): Welfare State Reform in Southern Europe. Fighting Poverty and Exclusion in Italy, Spain, Portugal and Greece, London, S. 1-32.

Frazer, H./Marlier, E. (2016): Minimum Income Schemes in Europe. A Study of National Policies 2015, European Commission. European Social Policy Network (ESPN), Brüssel.

Goedemé, T./Marchal, S. (2016): Exploring a blind spot in comparative pension reform research: long-term trends in non-contributory pensions in Europe, in: International Journal of Social Welfare 25(2), S. 161-175. 
Hubl, V./Pfeifer, M. (2013): Categorical differentiation in the light of deservingness perceptions: institutional structures of minimum income protection for immigrants and for the disabled, in: Marx, I./Nelson, K. (Hrsg.): Minimum Income Protection in Flux, Houndmills/Basingstoke, S. 108-136.

ILO = International Labour Organization (1942): Approaches to social security: an international survey, Studies and Reports, Series M, No. 1, Geneva.

Korpi, W./Palme, J. (1998): The paradox of redistribution and the strategy of equality: Welfare state institutions, inequality and poverty in the Western countries, in: American Sociological Review 63(5), S. 661-87.

Leibfried, S. (1992): Towards a European welfare state? On integrating poverty regimes into the European Community, in: Ferge, Z./Kolberg, J. E. (Hrsg.): Social Policy in a Changing Europe, Frankfurt am Main, S. 245-279.

Leisering, L. (2019): The Global Rise of Social Cash Transfers. How States and International Organizations Constructed a New Instrument for Combating Poverty, Oxford.

Marchal, S./Marx, I./van Mechelen, N. (2014): The great wake-up call? Social citizenship and minimum income provisions in Europe in times of crisis, in: Journal of Social Policy 43(2), S. 247-267.

Marx, I./Nelson, K. (2013): A new dawn for minimum income protection?, in: Marx, I./Nelson, K. (Hrsg.): Minimum Income Protection in Flux, Houndmills/Basingstoke, S. 1-27.

Matsaganis, M. (2017): The impact of the great recession on child poverty in Greece, in: Cantillon, B./Chzhen, Y./Handa, S./Nolan, B. (Hrsg.): Children of Austerity. Impact of the Great Recession on Child Poverty in Rich Countries, Oxford, S. 94-117.

Nelson, K. (2010): Social assistance and minimum income benefits in old and new EU democracies, in: International Journal of Social Welfare 19(4), S. 367-78.

Nelson, K. (2012): Counteracting material deprivation: the role of social assistance in Europe, in: Journal of European Social Policy 22(2), S. 148-163.

Neumann, F. (2016): Soziale Mindestsicherung in Europa. Leistungsprofile im Vergleich, Berlin.

OECD (2021): Adequacy of Minimum Income Benefits (table), www.oecd.org/ els/benefits-and-wages-statistics.htm (Abruf am 19.3.2021).

van Mechelen, N./Marchal, S. (2013): Struggles for life: social assistance benefits. 1992-2009, in: Marx, I./Nelson, K. (Hrsg.): Minimum Income Protection in Flux, Houndmills/Basingstoke, S. 28-53. 
